\title{
Application of microspectral luminescent analysis to study the intracellular metabolism in single cells and cell systems
}

\author{
Natalia A. Karnaukhova*, Larisa A. Sergievich, Valery N. Karnaukhov \\ Institute of Cell Biophysics, Russian Academy of Science, Pushchino, Russian Federation; ${ }^{*}$ Corresponding Author: $\underline{\text { nakarnaukhova@,mail.ru }}$
}

Received 4 December 2009; revised 27 January 2010; accepted 15 March 2010.

\begin{abstract}
Spectral luminescent analysis of single cells and cellular systems enable us to reveal the initial changes of intracellular metabolism that can followed by human diseases or failure in biocenosis. Two cytodiagnostic systems of devices and techniques have been developed: 1) Microspectrofluorimeters registering the fluorescent spectra of individual cells or intracellular organelles used for fundamental investigations of cell reactions and for discovering and studying new dimensionless fluorescent characteristic parameters reflecting the biochemical or physiological properties of the cells; 2) Double- and multi-wave microfluorimeters for rapid registration of fluorescent characteristic parameters for many cells to obtain statistical information about cell population. These techniques are useful especially in medical and ecological investigations.
\end{abstract}

Keywords: Intracellular Metabolism; Spectral Luminescent Analysis; Microspectrofluorimeters; Double and Multi-Wave Microfluorimeters; Cytodiagnostics; Low-Frequency Variable Magnetic Fields; Solar Activity

\section{INTRODUCTION}

All cells have a general physical-chemical basis of functioning in spite of different morphological structures and functions (synthesis of nucleic acids and proteins, energetic, etc.). Therefore, the success in the solution of this problem is essentially dependent on finding specific features which could form the basis of the algorithm for recognition of cells by their chemical composition. Besides, it is necessary to provide possibility for the morphological analysis of "suspicious" cells. The characteristic features common for cells of different kinds were defined on the base of fundamental studies of intracellular regulation of metabolism with microspectral luminescent analysis. It formed a new trend in automation of cytodiagnostics. To study plant, animal and microorganism cells both self-luminescence of some intracellular compounds (NADH, flavoproteins, for example) and the secondary luminescence induced by interaction of fluorochromes with the cells are used. The advance in fluorescent cytodiagnostics is only possible with best methods and tools available for microspectral analysis which present a combination of a fluorescent microscope with a spectroanalysing instrument supplied with electronic registration and control units. Such a system satisfying all needs of cytodiagnostics would consist of the two types of instruments. The interconnection between them is determined by their functional peculiarities which will be considered below with some characteristic parameters using in medicine and ecology [1-3].

\section{DEVICES AND TECHNIQUES FOR LUMINESCENT CYTODIAGNOSTICS}

\subsection{Microspectrofluorimeter- Microspectrophotometer}

This instrument is used to study luminescent spectral characteristics of cell. The general diagram of microspectrofluorimeters is given in Figure 1. This scheme can be varied depending on concrete task. Different dispersing elements are used as monochromator: prisms, diffraction gratings and interfering light filters of varying wave length [1]. Microspectrofluorimeter MSF-1 enables registration of luminescence spectra of cells and intracellular compartments up to $0.5 \mu \mathrm{m}$ in diameter in the range from 400 to $800 \mathrm{~nm}$. The luminescence spectra of individual cells are insufficient to follow any process, and information on the behavior of the whole cell population under study is required. It is convenient for this purpose to describe the luminescence spectrum of each cell with one parameter only. Thus, microspectrofluo- 
rimeters registering the fluorescent spectra of individual cells or intracellular organelles are used for fundamental investigations of cell reactions and for discovering and studying of the new dimensionless fluorescent characteristic parameters, reflecting the biological or physiological properties of the cells.

\subsection{Microfluorimeter DMF-2}

Using microspectrofluorimeter the luminescence spectra of various types of cells have been studied and the characteristic parameters of the cells have been defined, there is no need to register the total spectrum for each cell. It is enough to measure the luminescence intensity of the cells in two characteristic spectral regions. Microfluorimeter DMF-2 (Radical) interfaced to a PC/AT compatible computer is used to measure fluorescence intensities at two separate wavelengths (Figure 2). It is based on a fluorescent microscope with a double-channel fluorescent sensor assembly. The first channel is tuned to record one fluorescence intensity and the second another fluorescence intensity of the same object. This allows measurement of the fluorescent characteristics of definite single cells or their compartments, the structure of which is controlled by microscopy. The fluorescence of the cells is excited by the emission of a DRSh-250-2 mercury arc lamp with chosen wavelength. The size of the photometered area corresponds to the cell size. A special program "Microfluor" made it possible to obtain the distribution histograms of the fluorescence intensities in the different regions of the spectrum as well as the distribution histograms of the characteristic parameters for 200 cells within $15-20 \mathrm{~min}$ with the points plotted onto the phase plane, and to perform statistical analysis of the data $[4,5]$. These techniques are useful especially in medical and ecological investigations.

\section{APPLICATIONS OF DEVICES AND METHODS FOR LUMINESCENT CYTODIAGNOSTICS}

\subsection{Synthetic Activity of Cells}

To investigate synthetic activity of cells the characteristic parameter $\alpha$ have been offered. Fluorochrome acridine orange $(\mathrm{AO})$ is widely used to investigate the nucleic acids in living as well as fixed cells. As an example of information obtained with the help of MSF-1 it can consider fluorescence spectra of AO stained blood lymphocytes of rabbit at different stages of their immune response to exogenous protein ovalbumin [6]. The spectra consist of two emission bands. AO interacts with DNA and RNA by intercalation or electrostatic attraction respectively. DNA (double-helical nucleic acids) intercalated AO fluoresces green $(530 \mathrm{~nm})$, RNA (single- helical nucleic acids) electrostatically bound AO fluoresces red $(640 \mathrm{~nm})$.

Using the fluorescent microscope, investigator visually observes the cells with colors from green through yellow, orange to red in depend of ratio in emission bands only in two spectral diapasons-in green $\left(I_{530}\right)$ and red $\left(I_{640}\right)$. And if the color in cell fluorescence can't be

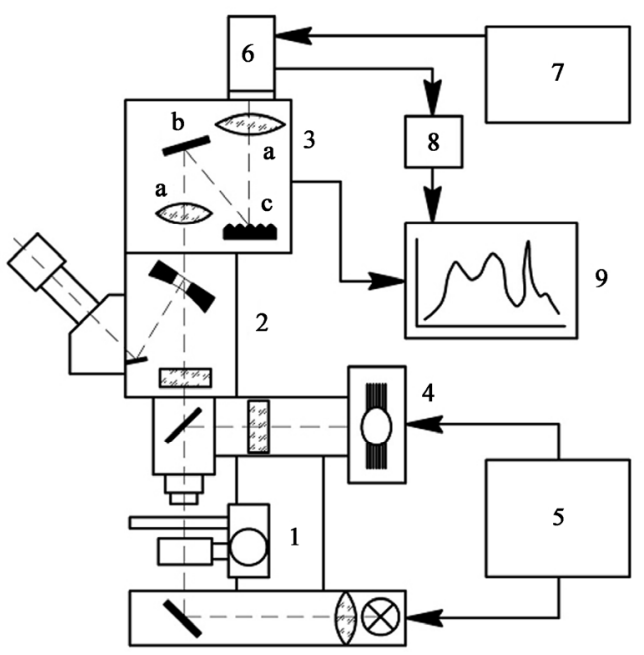

Figure 1. General scheme of MSF-1. 1-luminescent microscope; 2-probe nozzle; 3-system of monochromator: a-objective, b-mirror, c-diffraction grating; 4mercury arc lamp (DRSh-250-2); 5-power supply unit; 6-photomultiplier; 7-high voltage power unit; 8-amplifier; 9-register (X, Y-recorder).

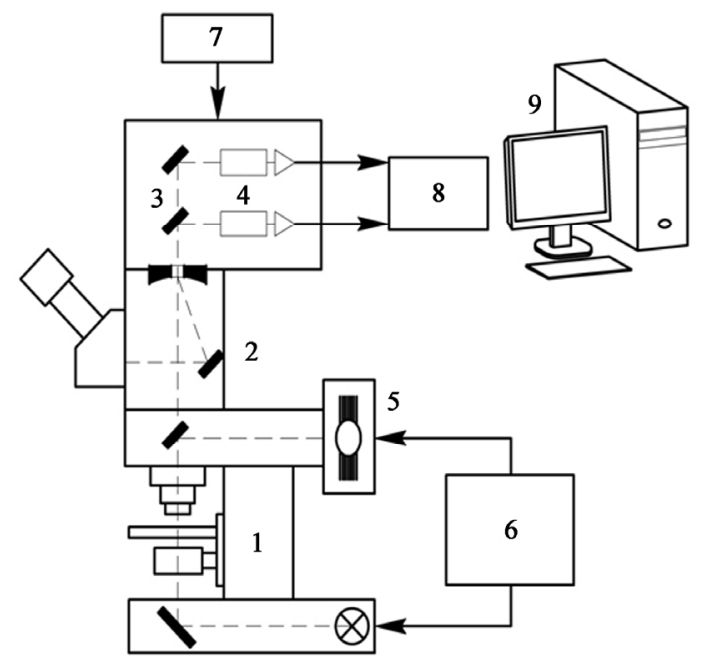

Figure 2. General scheme of two-channel (doublewave) microfluorimeter "Radical DMF-2". 1-luminescent microscope LUMAM; 2-probe nozzle; 3-dichroic mirror-analyzer; 4-two-channel (double- wave) registration system;5-mercury arc lamp (DRSh-2502); 6-power supply unit for mercury arc lamp; 7power supply unit for photomultipliers; 8-ADC; 9computer. 
used for analytical purpose, the description of cell's color as a ratio of fluorescence intensities.

$$
\alpha=\frac{I_{640}}{I_{530}}=A_{1} \frac{\left[N A_{1}\right]}{\left[N A_{2}\right]}
$$

where $A_{1}$ is the proportionality coefficient, [NA $]$ and $\left[N A_{2}\right]$ are the concentrations of single-helical $\left(N A_{1}\right)$ and double-helical $\left(N A_{2}\right)$ nucleic acids, permits to analyze quantitatively the processes in the cells.

Taking into account that in differentiated nonprolifirating cells (blood lymphocytes) the main quantity of single-helical nucleic acids is $R N A$, double-helical nucleic acids is $D N A$, expression (1) should be refined.

$$
\alpha=\frac{I_{640}}{I_{530}}=A_{1} \frac{[R N A]}{[D N A]}
$$

It has been shown that under definite conditions of cell staining with $A O$ parameter $\alpha$ reflects the amount of RNA per unit $D N A$ and, hence, characterizes cell synthetic activity [1-3,6]. Series of $\alpha$-distribution histograms is indicative of changes in synthetic activity of all population of immunocompetent cells at different stages of processes in immune system (Figure 3).

The above example demonstrates only one way of determining the parameter characterizing the ratio between the nucleic acids in the cell and the synthetic potentiality of the cell. Being, presumably, of greatest interest to medicine and biology [6-10], it is not the only possible method. Using the microspectral analysis of cells and a large set of luminescent dyes-labels, other useful characteristic parameters can be found. We further elaborate on our method for monitoring the synthetic activity of lymphocytes, testing the two-dye, three-color assay with regard to the temporal organization of the immune response. Within the same methodological framework, a good probe for protein is 1-anilino-8-naphthalene sulfonate $(A N S)$; we have already shown that consecutive staining of fixed cells with $A O$ and $A N S$ adds a third fluorescence peak in the blue region $(470 \mathrm{~nm})$ characteristic of protein-bound ANS [11].

$$
\beta=A \frac{[\text { protein }]}{[D N A]}=\frac{I_{470}}{I_{530}-0.4 \cdot I_{470}}
$$

The investigations conducted on blood lymphocytes showed that parameter $\alpha$ is sensitive to the action of the stimulatory and damaging effects of environmental factors, including electromagnetic fields and solar activity $[12,13]$.

Under the action of low-frequency variable magnetic fields with the specified parameters, the synthetic activity (parameter $\alpha$ ) increased by 22 to 35\% (Figure 4). These results and the data obtained by another methods showed that the variable magnetic fields enhances the synthetic activity of lymphocytes, improves the type of adaptive response, and thereby increases the level of the immune resistance of the organism.

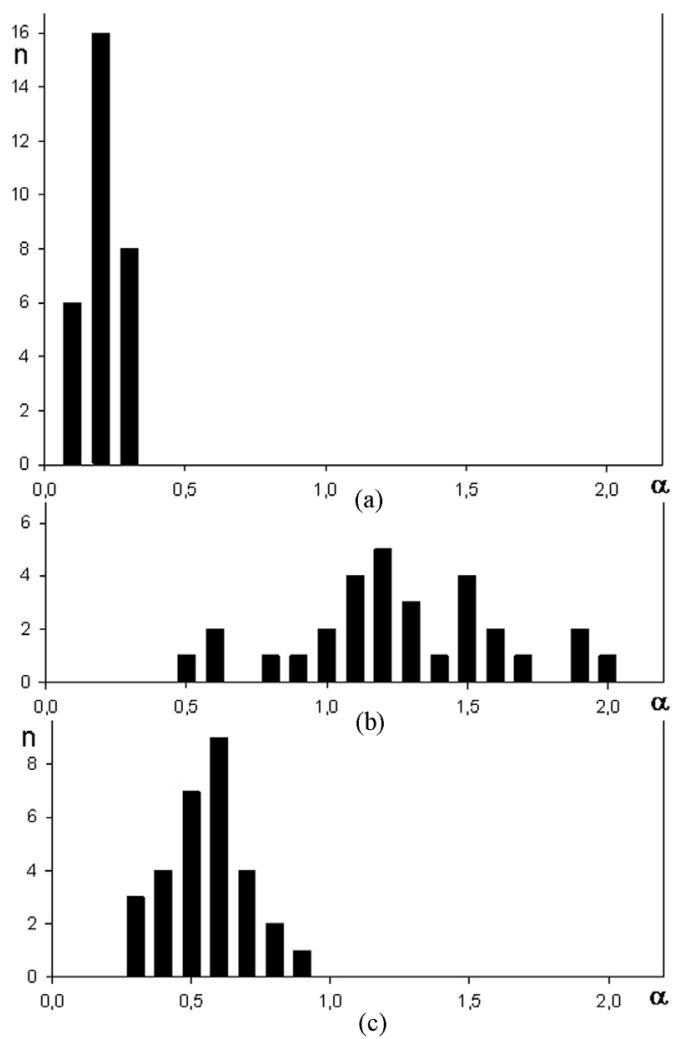

Figure 3. Distributions histograms of the parameter $\alpha$ for rabbit blood lymphocytes in process of immune response in organism onto albumin introduction: (a) before immunization; (b) on stage of the maximum activity; and (c) on the later stage. Along the ordinate is the number of cells. Along the abscissa are the $\alpha$ parameter values.

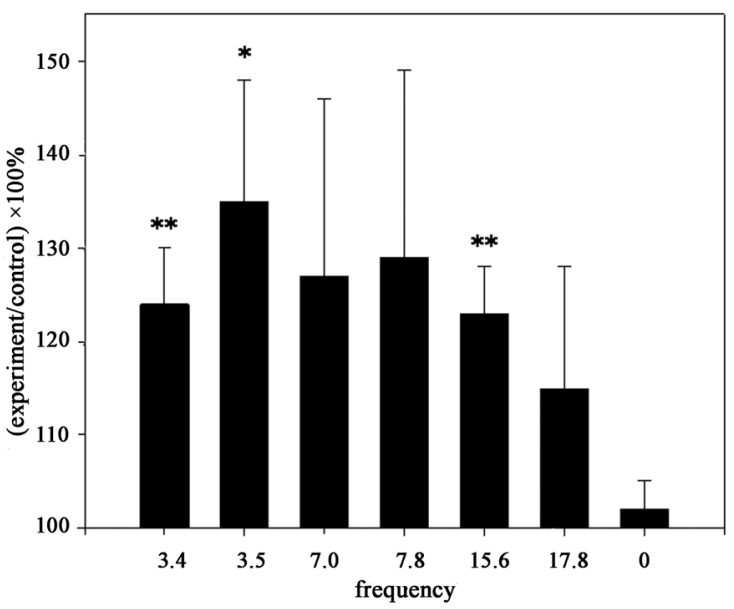

Figure 4. Diagrams of the changes in the average values of the parameters $\alpha$ for lymphocytes in the blood of rats as a function of frequency. ${ }^{*} \mathrm{p}<0.05,{ }^{* *} \mathrm{p}<0.01$. 
Correlation was revealed between solar activity parameters (sunspot number and the $10.7 \mathrm{~cm}$ solar radio flux) and the synthetic activity of blood lymphocytes in different species of animals during the same periods (January-April) of 1993, 1994, 2000, and 2002 (Table 1).

In the others seasons (April-June, August-December), the negative correlation between the studied indices was weaker and was not reliable. The data suggest seasonal regularities in the connection between the studied processes.

Sign reversal of the correlation coefficient was observed in the second maximum of solar cycle 23 . The change of sign may depend on a change in the ratio between phases of the oscillatory processes studied. However, the mechanism of the phenomenon observed remains unknown.

It was also shown that the correlation decreased under stronger internal (disease) or external (chronic gammairradiation) influences (Table 2).

\subsection{Energetics of Animal Cells}

Also the self-luminescence of living cells can be used for cytodiagnostics. For instance, it was shown $[1,14,15]$ that the state of the mitochondrial energetic apparatus of a cell can be quantitatively characterized by the ratio of the intensities of luminescence of oxidized flavoproteins $(530 \mathrm{~nm})$ and reduced pyridinenucleotides $(470 \mathrm{~nm})$ :

$$
\xi=\frac{I_{530}-0.5 \cdot I_{470}}{I_{470}}
$$

In this case the application of the microspectral analysis enabled one to find that in one cell (neurons of stretch-receptor) there are two mitochondrial pools responsible for the energy supply of different functional mechanisms of the cell-membrane transport and synthetic processes (Figure 5). It was shown that these two mitochondrial pools are controlled by different mechanisms. Therefore, in one and the same cell, at one and the same instant the mitochondria belonging to different

Table 1. Correlation coefficients between the $10.7 \mathrm{~cm}$ solar radio flux and the synthetic activity of blood lymphocytes in different years.

\begin{tabular}{llll}
\hline 1993 & 1994 & 2000 & 2002 \\
\hline$-0.81 * * \pm 0.08$ & $-0.63 * \pm 0.17$ & $-0.63 * \pm 0.15$ & $0.71 * \pm 0.09$ \\
\hline$* \mathrm{p}<0.05, * * \mathrm{p}<0.01$ & &
\end{tabular}

Table 2. Correlation coefficients between the $10.7 \mathrm{~cm}$ solar radio flux and the synthetic activity of blood lymphocytes in health, in pathology, and after gamma-irradiation.

\begin{tabular}{ccc}
\hline $\begin{array}{c}\text { Control } \\
\text { (healthy state) }\end{array}$ & $\begin{array}{c}\text { Pathology } \\
\text { (cholelithiasis) }\end{array}$ & $\begin{array}{c}\text { Gamma - irradiation (14.4 } \\
\text { cGy/day to 15 Gy) }\end{array}$ \\
\hline$-0.63 * \pm 0.17$ & $-0.24 \pm 0.26$ & $-0.45 \pm 0.22$ \\
\hline $\mathrm{p}<0.05$ & &
\end{tabular}

pools may be in different states of activity. It is only the method of spectral analysis that enables one to obtain such information about the state and organization of intracellular organelles in a functioning cell [1].

Taking into consideration the changes in the energetic apparatus of cells upon malignization found by Warburg it can be supposed that the characteristic parameter can also be used for automatic detection of cancerous malignant cells in preparation [16].

$$
\xi_{1}=\frac{A}{B}=\frac{I_{530}}{I_{470}}
$$

\subsection{Ecology and Environment Protection.}

In solving the problems of environment protection, the relationship between autotrophic and heterotrophic energy provision reflects the wellbeing of an autotrophic organism [2]. The energetic state of high plant cells can be determined from the ratio luminescence band intensities of chlorophyll $(680 \mathrm{~nm})$ and oxidized flavoproteins $(530 \mathrm{~nm})$.

$$
X=\frac{A}{B}=\frac{I_{680}}{I_{530}}
$$

This makes it possible to detect regions with polluted atmosphere, to assess the state of the forests as well as to predict the harvest of cultivated plants $[2,17]$. For the same purpose, the following parameters can also be used.

$$
\begin{aligned}
& \varphi=\frac{A}{B}=\frac{I_{680}}{I_{645}} \\
& \psi=\frac{A}{B}=\frac{I_{680}}{I_{572}}
\end{aligned}
$$

Those characterize the state of lichens symbiotic

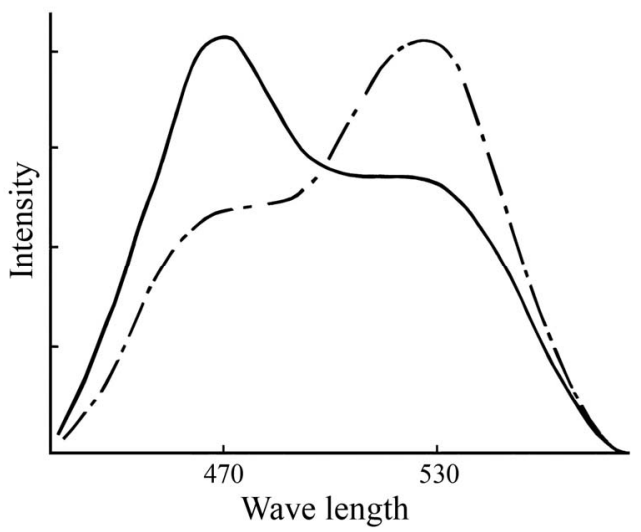

Figure 5. Luminescence spectra of reduced pyridinenucleotides (NADH, $470 \mathrm{~nm}$ ) and oxidized flavoproteins $(\mathrm{FPo}, 530 \mathrm{~nm})$ in neuron of stretch-receptor. Ordinate-fluorescence intensity in rel. units. Abscissa-the wave length in $\mathrm{nm}$. 
blue-green algae which are extremely sensitive to atmosphere pollution in places of their habitat. In the both cases, the ratio of luminescence band intensity of chlorophyll $(680 \mathrm{~nm})$ to that of phycocyanine $(645 \mathrm{~nm})$ or phycoerythrin $(572 \mathrm{~nm})$ is used [2]. It is clearly seen that the autotrophic cells having intense chlorophyll band in the luminescent spectrum (Figure 6, curve 1) later start to switch from autotrophic (algal) to heterotrophic (bacterial) energy provision (Figure 6, curves 2, 3).

It is important that in the tropical Atlantic the majorities of the Cyanophyceae contain no chlorophyll and are actually heterotrophic cyanobacteria. Their typical fluorescence spectra (Figure 7, curve 1) have only phycoerythrin $(572 \mathrm{~nm})$ and phycocyanine $(645 \mathrm{~nm})$ bands, though there are occasional cells that have not completely lost chlorophyll (Figure 7, curves 2, 3).

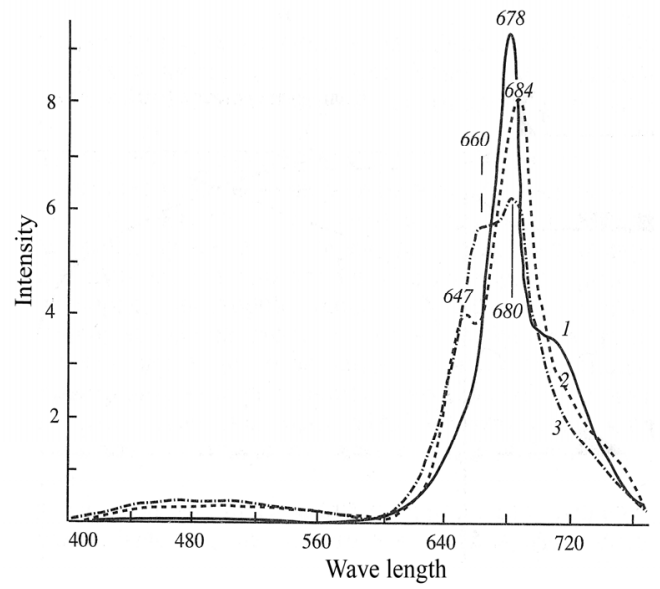

Figure 6. Luminescence spectra of single cells of blue-green microorganisms in different states: 1autotrophic (algal); 2, 3-mixed (autotrophic and heterotrophic) energy provision.

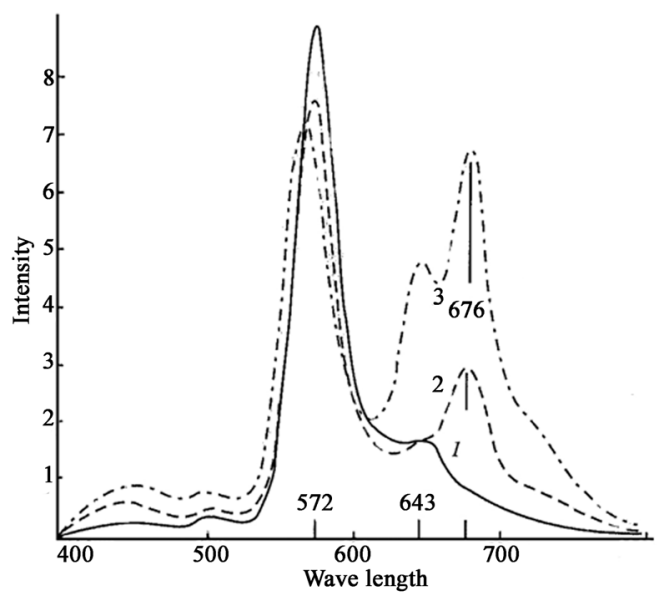

Figure 7. Luminescence spectra of blue-green microorganisms in the tropical Atlantic: 1-a most typical spectrum, 2, 3-deviant spectra.
However, most interesting seems the application of the parameters $\varphi$ and $\psi$ in the cases when it is necessary to predict the onset of "blooming" of blue-green algae in water storage basins or lakes $[18,19]$ for determining the optimal instant of treating algae population with bacteriophages (cyanophages) in order to prevent "blooming" which causes disastrous effect. Of particular importance may be the application of the parameters $\varphi$ and $\psi$ for predicting the initiation of the disease "ciguatera". It is the poisoning of people with usually harmless fishes and mollusks living in regions of intense "blooming" of blue-green algae in tropic zones of the oceans [19].

\section{CONCLUSIONS}

These few examples indicate that the methods of luminescent cytodiagnostics have promising applications in different fields of biology, medicine, environment protection and biotechnology. The realization of these perspectives necessitates the development of special equipment. Two cytodiagnostic systems of devices and techniques have been used for the purpose:

1) Microspectrofluorimeters-microspectrophotometers registering the fluorescent spectra of individual cells or intracellular organelles are used for fundamental investigations of cell reactions and for discovering and studying of the new dimensionless fluorescent characteristic parameters, reflecting the biochemical or physiological properties of the cells.

2) Double-and multi-wave microfluorimeters are used for the rapid registration of many cells to obtain statistical information about cell population. These techniques are useful especially for medical and ecological investigations.

Thus, this study confirms that parameters derived from luminescent spectral analysis of fluorochromed cells (such as $\alpha$ and $\beta$ ) or self-luminescence of living cells (such as $\xi, \chi, \varphi, \psi$ ) are valid as dynamic indices of intracellular metabolic activity in single cells and cell systems. There are broad possibilities of further developing this approach and equipment into developing increasingly differentiated fluorescence techniques.

\section{REFERENCES}

[1] Karnaukhov, V.N. (1978) Luminescent spectral analysis of cell. Nauka, Moscow.

[2] Karnaukhov, V.N. (2001) Spectral analysis in cell-level monitoring of environmental state. Nauka, Moscow.

[3] Karnaukhov, V.N. (1978) Luminescent analysis of cell. Nauka, Moscow. http://www.edu.ru/db/ portal/e-library/ 00000048/00000048.htm

[4] Karnaukhov, V.N., Yashin, V.A., Karnaukhova, N.A., Kazantsev, A.P. and Karnaukhov, A.V. (1999) Double-wave microfluorimeter "Radical DMF-2". Book of Abstracts II Congress of Biophysicists of Russia, 2, 
594-595.

[5] Karnaukhova, N.A., Sergievich, L.A., Kuzhevskij, B.M. Sigaeva, E.A., Nechaev, O.Y., Karnaukhov, V.A. and Karnaukhov, V.N. (2007) A study of the correlation between the functional activity of blood lymphocytes in different animals and intensity of neutrons near the earth surface. Biophysics, 52(4), 699-704.

[6] Karnaukhova, N.A. (1984) Luminescence parameters of blood nuclear cells in process of immune reaction in organism. Biophysics, 29(2), 276-279.

[7] Karnaukhova, N.A. (1991) Changes in fluorescent spectra of acridine orange stained blood cells from patient suffering from lymphosarcoma and leukemias in the course of chemotherapy. Experimental Oncology, 13(1), 50-53.

[8] Gordon, R.Y., Bocharova, L.S., Kruman, I.I., Popov, V.I., Kazantsev, A.P., Khutzian, S.S. and Karnaukhov, V.N. (1997) Acridine orange as an indicator of ribosome state in cell. Cytometry, 29(3), 215-221.

[9] Karnaukhova, N.A., Sergiyevich, L.A., Aksenova, G.E. and Karnaukhov, V.N. (1999) Synthetic activity of rat blood lymphocytes under acute and continuous gamma irradiation-fluorescent microspectral study. Radiation and Environmental Biophysics, 38(1), 49-56.

[10] Karnaukhova, N.A., Lubet, P.E., Katania, R., Karnaukhov, A.V., Sergievich, L.A. and Karnaukhov, V.N. (2003) Microspectral studies on neuroendocrine regulation of gametogenesis in mollusk. Biophysics, 48(5), 869-873.

[11] Karnaukhova, N.A., Sergiyevich, L.A. and Karnaukhov, V.N. (2008) Dinamics of ribosomal activity and protein production in peripheral blood lymphocytes during an immune response. Biophysics, 53(4), 632-637.

[12] Karnaukhova, N.A., Sergievich, L.A., Kvakina, E.B., Barsukova, L.P., Mar'yanovskaya, G.Y. and Kuz'-menko, T.S. (2000) Study into the changes in the functional state of the synthesis apparatus of blood lymphocytes under the action of weak low-frequency magnetic fields. Biophysics, 45(4), 697-703.

[13] Karnaukhova, N.A., Sergievich, L.A., Karnaukhov, V.A. and Karnaukhov, V.N. (2004) Changes in the synthetic activity of lymphocytes under the action of physical factors related to Solar activity variations. Biophysics, 49(suppl.1), 552-559.

[14] Karnaukhov, V.N., Lebedev, O.E. and Pavlenko, V.K. (1976) About two mitochondrial pools in a stretch-receptor neuron. Tsitologia, 18(10), 1189-1193.

[15] Rudenko, Y.N., Bigdai, E.V. and Samoilov, V.O. (2007) Kinetics of $\mathrm{Ca}^{2+}$, NADH and oxidized flavoproteids in the frog olfactory living under the effect of odorants. Biophysics, 52(1), 88-94.

[16] Thorell, B. (1981) Flow cytometric analysis of cellular endogenous fluorescence. Cytometry, 2(1), 39-43.

[17] Roshchina, V.V. (2003) Autofluorescence of plant secreting cells as a biosensor and bioindicator reaction. Journal of Fluorescence, 13(5), 403-420.

[18] Karnaukhov, V.N., Martsenuk P.P. and Yashin, V.A. (1980) Luminescence spectral characteristics of physiological state of cells of blue-green algae. Fisiologia Rast, 27(1), 11-17.

[19] Karnaukhov, V.N. and Yashin, V.A. (2003) Spectral studies on single cells of sea microplankton: History and prospects. Biophysics, 48(5), 940-949. 